\title{
Feeding Activated Charcoal from Bark Containing Wood Vinegar Liquid (Nekka-Rich) Is Effective as Treatment for Cryptosporidiosis in Calves
}

\author{
S. Watarai, ${ }^{\star 1}$ Tana, $\dagger$ and M. Koiwał \\ *The Laboratory of Veterinary Immunology, Division of Veterinary Science, Graduate School of Life and Environmental Sciences, \\ Osaka Prefecture University, 1-1 Gakuen-cho, Naka-ku, Sakai, Osaka 599-8531, Japan \\ †Research Institute, Miyazaki-Midori Pharmaceuticals Inc., 945 Hieda, Akae, Miyazaki 880-0912, Japan \\ fDepartment of Veterinary Internal Medicine, Veterinary Teaching Hospital, School of Veterinary Medicine, Rakuno Gakuen University, \\ 582 Bunkyodai-Midorimachi, Ebetsu, Hokkaido 069-8501, Japan
}

\begin{abstract}
The effect of activated charcoal containing wood vinegar liquid (Nekka-Rich) on Cryptosporidium parvum was evaluated in vitro and in vivo. First, the adsorption of $C$. parvum by the activated charcoal component of Nekka-Rich was examined. When mixed, C. parvum oocysts were effectively adsorbed by activated charcoal. Next, the effect of the wood vinegar liquid component of Nekka-Rich was assessed. Wood vinegar liquid had an antiprotozoan activity against $C$. parvum oocysts. Finally, the anticryptosporidial effect of Nekka-Rich was evaluated in calves experimentally infected with C. parvum. Six neonatal calves at $7 \mathrm{~d}$ of age were orally infected with $1 \times 10^{5}$ oocysts of $C$. parvum. When diarrhea was observed (on d 3 after the experimental infection), 3 calves received a milk replacer containing $10 \mathrm{~g}$ of NekkaRich at 8-h intervals for 4 consecutive days, and 3 control calves were given a milk replacer without Nekka-Rich at 8-h intervals for 4 consecutive days. Calves fed milk with Nekka-Rich showed recovery from diarrhea $1 \mathrm{~d}$ after the start of treatment. Significantly less fecal excretion of $C$. parvum oocysts was observed $1 \mathrm{~d}$ after treatment in calves fed Nekka-Rich. On d 2 from the beginning of treatment with Nekka-Rich (on d 5 after the experimental infection), oocysts were not isolated from fecal samples. On the other hand, nontreated control calves developed severe diarrhea, and fecal excretion of oocysts was consistently observed. Calves in both groups were killed for collection of samples, which were subjected to scanning electron microscopy. Scanning electron microscopy revealed adherence of $C$. parvum oocysts to the intestinal epithelial surface of nontreated control calves, whereas a lack of $C$. parvum adhesion was observed in calves fed Nekka-Rich. These results suggest
\end{abstract}

Received June 1, 2007.

Accepted December 18, 2007.

${ }^{1}$ Corresponding author: swatarai@vet.osakafu-u.ac.jp that Nekka-Rich would be useful for the treatment of cryptosporidiosis in neonatal calves.

Key words: activated charcoal, cryptosporidiosis, diarrhea, wood vinegar liquid

\section{INTRODUCTION}

Cryptosporidium parvum is an intestinal protozoan parasite that causes diarrhea in both humans and domestic animals (Casemore et al., 1997; Anderson, 1998; Xiao et al., 2004). In livestock, C. parvum infection in calves is of clinical and economic concern for producers (Casemore et al., 1997). Because the feces of calves are a recognized source of Cryptosporidium, fecal contamination of drinking water is a potential means of transmission of this protozoan (Smith, 1998; Fayer et al., 2000). Thus, methods for controlling C. parvum infection in calves are needed not only to reduce the clinical and economic impacts on the producer, but also to alleviate environmental and public health concerns.

Activated charcoal is known as a universal adsorbent because it can bind with variety of molecules (Chandy and Sharma, 1998). It has been reported that activated charcoal is useful for the removal of bacteria and bacterial toxins, both in vitro and in vivo (Drucker et al., 1977; Pegues et al., 1979; Du et al., 1987; Gardiner et al., 1993). Recently, we have shown the usefulness of activated charcoal for removal of verotoxin-producing Escherichia coli and verotoxin (Naka et al., 2001). Others have reported that organic acids inhibit the growth of enteropathogenic bacteria (Anderson, 1992; Hsiao and Siebert, 1999; Nakai and Siebert, 2003) as well as the viability of C. parvum (Kniel et al., 2003). Nekka-Rich (MiyazakiMidori Pharmaceuticals Inc., Miyazaki, Japan) is a product made by mixing activated charcoal and wood vinegar liquid that contains organic acids. The activated charcoal and wood vinegar liquid of Nekka-Rich are both obtained from the bark of evergreen oak (Castanopsis cuspidata and Quercus acuta) by carbonization.

Based on a recent study in our laboratory that demonstrated that Nekka-Rich effectively inhibits the infection 
of chickens with Salmonella enterica serovar Enteritidis (Watarai and Tana, 2005), we hypothesized that NekkaRich may be useful for the prevention of $C$. parvum infection in calves by inhibiting adsorption of oocysts and reducing the viability of oocysts. Therefore, we examined each component of Nekka-Rich for activity in vivo against $C$. parvum and evaluated the combined commercial product in neonatal calves experimentally infected with $C$. parvum. In addition, we also evaluated the ability of Nekka-Rich to inhibit intestinal colonization of $C$. parvum in neonatal calves experimentally infected with C. parvum.

\section{MATERIALS AND METHODS}

\section{Materials and Parasites}

Activated charcoal and wood vinegar liquid, which are obtained from the bark of evergreen oak (C. cuspidata and $Q$. acuta), and Nekka-Rich (a product made from these 2 components) were obtained from Miyazaki-Midori Pharmaceuticals Inc. In Japan, Nekka-Rich is approved as a feed additive. Cryptosporidium parvum oocysts were collected from feces of calves with cryptosporidiosis by sucrose flotation method as described previously (Uga et al., 2000). Purified oocysts were used for in vitro and in vivo experiments.

\section{In Vitro Adsorption Test}

This study was designed to test the usefulness of activated charcoal from the bark of an evergreen oak for $C$. parvum adsorption. Cryptosporidium parvum oocysts (2 $\times 10^{4}$ oocysts) in $1 \mathrm{~mL}$ of PBS ( $\mathrm{pH} 7.2$ ) and activated charcoal from the bark of an evergreen oak (0, 1, 5, and $10 \mathrm{mg}$ ) were mixed together and incubated at $37^{\circ} \mathrm{C}$ for $1 \mathrm{~h}$ with gentle agitation. To remove the activated charcoal, the mixtures were passed through a membrane filter (Millipore, Bedford, MA; $10 \mu \mathrm{m}$ pore size), which was rinsed with $10 \mathrm{~mL}$ of PBS. The filtrates were centrifuged at $1,200 \times g$ for $10 \mathrm{~min}$, and the resulting supernatant was decanted. The remaining pellet of oocysts was suspended in PBS $(100 \mu \mathrm{L})$ and the number of oocysts was counted 3 times in a hemocytometer.

\section{In Vitro Effect of Wood Vinegar Liquid on C. parvum Oocyst}

Cryptosporidium parvum oocysts $\left(6 \times 10^{4}\right.$ oocysts $)$ were added to $1 \mathrm{~mL}$ of PBS containing $0,0.5,2.5$, and $5.0 \%$ wood vinegar liquid and incubated at $37^{\circ} \mathrm{C}$ for 14 $\mathrm{h}$ with gentle agitation. After incubation, each mixture of oocysts was centrifuged at $1,200 \times g$ for $10 \mathrm{~min}$ and the resulting supernatant was removed as described above.
Oocysts were resuspended in $100 \mu \mathrm{L}$ of PBS and counted 3 times in a hemocytometer.

\section{Animals}

Six male Holstein-Friesian calves that did not have natural infection with $C$. parvum were purchased at 3 $\mathrm{d}$ of age from 3 large local farms. Calves were housed individually in a biosafety level 2 isolation facility for large animals at Veterinary Teaching Hospital, School of Veterinary Medicine, Rakuno Gakuen University (Ebetsu, Japan). Calves were fed with a commercially available milk replacer (Scientific Feed Laboratory Co. Ltd., Tokyo, Japan) at intervals of $8 \mathrm{~h}$ for the duration of the experiment. They were maintained for the study in accordance with the guidelines of the Rakuno Gakuen University Committee on animal care and use.

\section{Experimental C. parvum Infection in Calves and Administration of Nekka-Rich}

Six calves were randomly assigned to 2 groups (3 calves/group). Physical contact between calves was prevented. All calves were inoculated orally with $1 \times 10^{5} \mathrm{C}$. parvum oocysts. At the onset of diarrhea, calves in the nontreated control group were given commercial milk replacer without Nekka-Rich, whereas calves in the Nekka-Rich-treated group received commercial milk replacer containing $10 \mathrm{~g}$ of Nekka-Rich at each feeding for $4 \mathrm{~d}$. All feces were examined microscopically for the screening of $C$. parvum infection. The clinical response of each calf was noted throughout the experiment in terms of presence and severity of diarrhea and oocyst shedding in stool. The presence of diarrhea was based on fecal consistency score using the following index: $1=$ normal feces; 2 = soft feces; $3=$ mild diarrhea; 4 = severe watery diarrhea. A daily sample of feces was collected from each calf from d 0 until $d 7$, and these samples were examined for the presence of $C$. parvum oocysts by the sucrose flotation method. In brief, $5.0 \mathrm{~g}$ of feces was mixed with $30 \mathrm{~mL}$ of distilled water; then, the fecal suspensions were passed through a stainless steel mesh sieve to remove solids and centrifuged at $300 \times g$ for 10 min. Each resulting supernatant was decanted and the sediment was resuspended in $14 \mathrm{~mL}$ of sucrose solution (specific gravity: 1.2). The suspension was transferred to a $15-\mathrm{mL}$ centrifuge tube and centrifuged at $300 \times \mathrm{g}$ for $10 \mathrm{~min}$. After centrifugation, the tube was left for 10 min. One drop of supernatant was then placed on a microscope slide, covered with an $18 \times 18 \mathrm{~mm}$ coverslip, and examined at 1,000 $\times$ magnification using a light microscope (Olympus Co., Tokyo, Japan). Fecal samples containing oocysts were categorized into 4 score groups as follows: 0: zero oocysts in all microscopic fields; $1:<10$ 
oocysts in 30 microscopic fields; 2 : $\geq 10$ to $<300$ oocysts in 30 microscopic fields; $3: \geq 300$ oocysts in 30 microscopic fields. Seven days after the experimental infection of $C$. parvum, all calves were humanely killed by excessive anesthetization in accordance with the guidelines of the Rakuno Gakuen University Committee on animal care and use, and sections of small intestines were collected to evaluate the degree of colonization of $C$. parvum.

\section{Scanning Electron Microscopy}

Scanning electron microscopy was used to evaluate the colonization of C. parvum. Tissue samples from mid duodenum, mid jejunum, and mid ileum were fixed in $3.0 \%$ glutaraldehyde in $0.1 M$ phosphate buffer ( $\mathrm{pH} 7.4)$ for $2 \mathrm{~h}$ at $4^{\circ} \mathrm{C}$ and then postfixed with $0.2 \%$ osmium tetraoxide in $0.1 \mathrm{M}$ phosphate buffer at room temperature for $1 \mathrm{~h}$. The tissue strips were dehydrated through a graded series of acetone, treated with $t$-butyl alcohol, dried in a freeze-drying apparatus (JFD-300, JEOL, Tokyo, Japan), coated with a 10-nm-thick gold layer in an ion coater (NL-OPC40, JEOL Datum Ltd., Tokyo, Japan), and examined with a scanning electron microscope (JSM600F, JEOL).

\section{Statistical Analysis}

Student's $t$-test was performed for statistical evaluation of in vitro results. Results are expressed as the arithmetic mean with the standard error of the mean (mean $\pm \mathrm{SEM})$. In addition, data were collected on the number of calves having diarrhea and the number of calves shedding oocysts for each treatment group for each day of data collection. These were compared between treatment groups by use of Fisher's exact test. The level of significance for all statistical tests was set at $P \leq 0.05$.

\section{RESULTS}

\section{Adsorption Effect of Activated Charcoal from the Bark of an Evergreen Oak on C. parvum}

As shown in Figure 1, activated charcoal from the bark of an evergreen oak caused a dose-dependent adsorption of $C$. parvum oocysts. That is to say, when $C$. parvum oocysts $\left(2 \times 10^{4}\right.$ oocysts) were incubated with $0,1,5$, and $10 \mathrm{mg}$ of activated charcoal at $37^{\circ} \mathrm{C}$ for $1 \mathrm{~h}$, the number of oocysts was $1.89 \pm 0.11 \times 10^{4}, 1.2 \pm 0.12 \times 10^{4}(P<$ $0.0125), 7.87 \pm 0.59 \times 10^{3}(P<0.001)$, and $4.43 \pm 0.3 \times$ $10^{3}(P<0.0003)$, respectively.

\section{Effect of Wood Vinegar Liquid on Oocysts}

As shown in Figure 2, the number of $C$. parvum oocysts was lowered in a dose-dependent manner by the addition

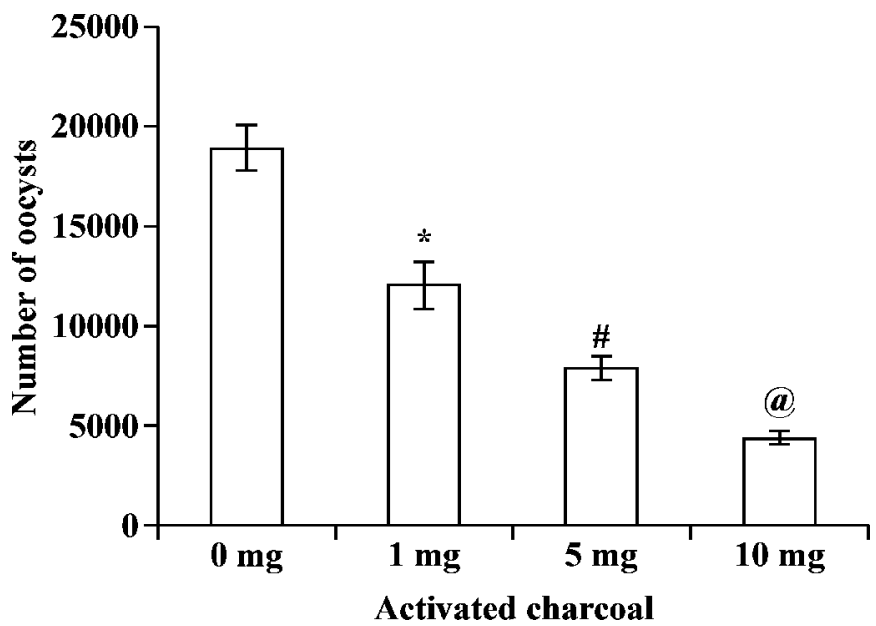

Figure 1. The capacity of activated charcoal to adsorb Cryptosporidium parvum. Data are expressed as mean $\pm \mathrm{SE}$ of triplicate experiments. ${ }^{*}=P<0.0125$, \# = P $<0.001, @=P<0.0003$ compared with control $(0 \mathrm{mg})$ (Student's $t$ test).

of wood vinegar liquid. On the other hand, the number of oocysts did not change when C. parvum was incubated with PBS without wood vinegar liquid.

\section{Clinical Response of Calves after Challenge Exposure with C. parvum Oocysts}

The clinical observations of calves after challenge with C. parvum oocysts and subsequent treatment with Nekka-Rich are shown in Table 1. All calves of the nontreated control group, when exposed to C. parvum oocysts, had mild (mean fecal consistency score 3.3) to

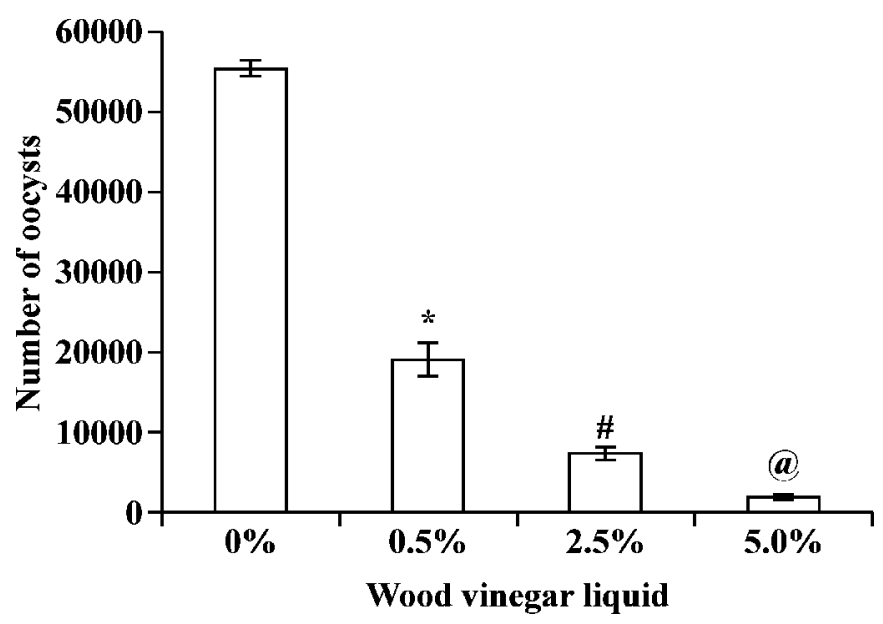

Figure 2. Effect of wood vinegar liquid on Cryptosporidium parvum. Data are expressed as mean \pm SEM of triplicate experiments. $*=P<0.0014$, \# = P<0.0006, @ = $P<0.0004$ compared with control (0\%) (Student's $t$ test). 
Table 1. Clinical response of calves to challenge exposure with Cryptosporidium parvum oocysts and treatment with Nekka-Rich on d 0 through 7

\begin{tabular}{lcccccccc}
\hline & \multicolumn{7}{c}{ Calves with diarrhea ${ }^{2}$ /total calves, $\mathrm{n} / \mathrm{n}$} \\
\cline { 2 - 8 } Group ${ }^{1}$ & 0 & 1 & 2 & 3 & 4 & 5 & 6 & 7 \\
\hline $\mathrm{A}$ & $0 / 3$ & $0 / 3$ & $0 / 3$ & $3 / 3$ & $3 / 3$ & $3 / 3$ & $3 / 3$ & $3 / 3$ \\
& $(1.0)$ & $(1.0)$ & $(1.0)$ & $(3.3)$ & $(3.3)$ & $(3.7)$ & $(3.3)$ & $(3.3)$ \\
$\mathrm{B}$ & $0 / 3$ & $0 / 3$ & $0 / 3$ & $3 / 3$ & $0 / 3^{*}$ & $0 / 3^{*}$ & $0 / 3^{*}$ & $0 / 3^{*}$ \\
& $(1.0)$ & $(1.0)$ & $(1.0)$ & $(3.3)$ & $(2.0)$ & $(2.0)$ & $(2.0)$ & $(2.0)$ \\
\hline & ${ }^{1} \mathrm{~A}=$ nontreated control calves; B = calves treated with Nekka-Rich. \\
2 Data in parentheses are mean fecal consistency scores: 1 = normal feces; 2 = soft feces; $3=$ mild diarrhea; \\
4 = severe watery diarrhea. \\
*P = 0.05 compared with group A (Fisher's exact test).
\end{tabular}

severe watery diarrhea (mean fecal consistency score 3.7) from postchallenge $d 3$ to 7 (the final day of experiment). However, there was a difference in fecal consistency score between the nontreated control group and the Nekka-Rich-treated group. Although calves of the treatment group developed mild diarrhea on postchallenge d $3(P=1.0)$, they had feces with a soft consistency within $1 \mathrm{~d}$ after feeding with commercial milk replacer containing Nekka-Rich (d 4: $P=0.05$ ), and no overt diarrheal disease was observed for the period when milk replacer containing Nekka-Rich was administered (d 5, 6, and 7: $P=0.05$ ).

\section{Presence of C. parvum Oocysts in Feces}

Table 2 shows the effect of Nekka-Rich-containing milk feeding on the suppression of $C$. parvum oocyst excretion in feces. On d 4 after challenge with C. parvum oocysts, the oocysts were isolated from fecal samples of all calves. However, there was a significant difference in fecal oocyst score between the nontreated control calves and calves treated with Nekka-Rich on d 5, 6, and 7 after challenge with $C$. parvum oocysts. The nontreated calves were excreting oocysts until the end of experiment period (d 7). On the other hand, no C. parvum oocysts were isolated from fecal samples of Nekka-Rich-fed calves $(P=0.05)$ from postchallenge d 5 to 7 , in contrast to the nontreated control calves.

\section{Detection of C. parvum Adherence in Small Intestines of Calves}

Figure 3 shows a scanning electron micrograph of ileal surfaces of nontreated control and Nekka-Rich-treated calves infected with $C$. parvum oocysts. The ileal section from nontreated control calves showed adherence of $C$. parvum on its surface. On the other hand, the ileal surface of Nekka-Rich-fed calves showed no C. parvum adhesion. Other intestinal sections such as duodenum and jejunum from calves treated with Nekka-Rich also revealed no adherent $C$. parvum oocysts along the intestinal epithelial surface, whereas adhering oocysts were present along the epithelial surface of the duodenum and jejunum of calves in the nontreated group (data not shown). These results are in accordance with those from C. parvum oocyst isolation shown in Table 2 .

\section{DISCUSSION}

We showed that activated charcoal of Nekka-Rich has adsorption efficacy against $C$. parvum. In general, activated charcoal has a high adsorptive capacity although

Table 2. Isolation (on d 0 through 7) of Cryptosporidium parvum oocysts from feces of challenged calves treated with or without Nekka-Rich

\begin{tabular}{lcccccccc}
\hline & \multicolumn{7}{c}{ C. parvum-positive calves ${ }^{2} /$ total calves } \\
\cline { 2 - 9 } Group $^{1}$ & 0 & 1 & 2 & 3 & 4 & 5 & 6 & 7 \\
\hline $\mathrm{A}$ & $0 / 3$ & $0 / 3$ & $0 / 3$ & $0 / 3$ & $3 / 3$ & $3 / 3$ & $3 / 3$ & $3 / 3$ \\
& $(0.0)$ & $(0.0)$ & $(0.0)$ & $(0.0)$ & $(1.0)$ & $(2.0)$ & $(2.0)$ & $(2.0)$ \\
$\mathrm{B}$ & $0 / 3$ & $0 / 3$ & $0 / 3$ & $0 / 3$ & $3 / 3^{*}$ & $0 / 3^{*}$ & $0 / 3^{*}$ & $0 / 3^{*}$ \\
& $(0.0)$ & $(0.0)$ & $(0.0)$ & $(0.0)$ & $(1.0)$ & $(0.0)$ & $(0.0)$ & $(0.0)$ \\
\hline
\end{tabular}

\footnotetext{
${ }^{1} \mathrm{~A}=$ nontreated control calves; $\mathrm{B}=$ calves treated with Nekka-Rich.

${ }^{2}$ Data in parentheses show oocyst score: 0 : zero oocysts in all microscopic field; $1:<10$ oocysts in 30 microscopic fields; 2 : $\geq 10$ to $<300$ oocysts in 30 microscopic fields; $3: \geq 300$ oocysts in 30 microscopic fields. $* P=0.05$ compared with group A (Fisher's exact test).
} 

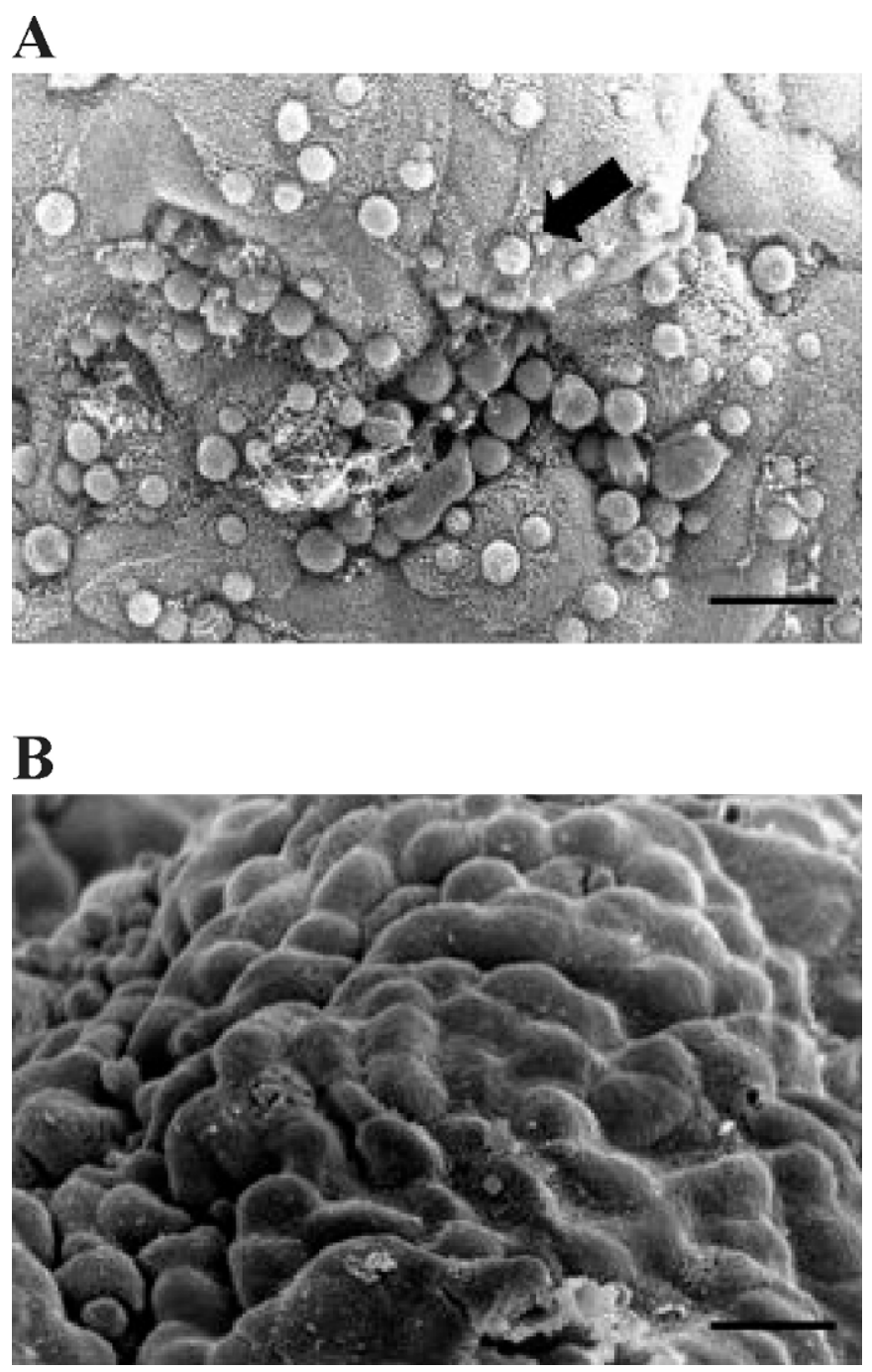

Figure 3. Electron micrographs of representative ileal sections: A) villi from the ileum of a nontreated calf showing adherent Cryptosporidium parvum oocysts (arrow); B) villi from the ileum of a calf treated with Nekka-Rich, showing the absence of adherent C. parvum oocysts. Bar $=10 \mu \mathrm{m}$.

it tends to be nonselective (Wicks et al., 1980; Chandy and Sharma, 1998). The adsorptive capacity of activated charcoal depends on its pore size. For removal of relatively large materials, an activated charcoal having larger pores is needed, whereas small substances need small pores (Chandy and Sharma, 1998). In this study, C. parvum oocysts were effectively adsorbed by activated charcoal from the bark of an evergreen oak, which suggests that this activated charcoal possessed pores of sufficient diameter for C. parvum oocysts. Indeed, the existence of various pores of $10 \mu \mathrm{m}$ or less in diameter was confirmed in the activated charcoal from the bark of an evergreen oak (unpublished data). More recently, we reported that activated charcoal from the bark of an evergreen oak has low binding capacity to Enterococcus faecium, which is smaller than C. parvum (Watarai and Tana, 2005). This suggests that oral administration of this activated charcoal could reduce $C$. parvum oocysts while minimizing the removal of normal bacterial flora in the intestinal tract.

We also found that the number of $C$. parvum oocysts was decreased by treatment with wood vinegar liquid. This suggests that wood vinegar liquid from the bark of an evergreen oak contains compounds with disinfectant activity against $C$. parvum oocysts. Wood vinegar liquid contains organic acids, which have been shown by others to inhibit the growth of enteropathogenic microbes (Anderson, 1992; Hsiao and Siebert, 1999; Nakai and Siebert, 2003) and to reduce the viability of C. parvum (Kniel, 2003). Thus, we believe the organic acids are responsible for the inhibitory effect on C. parvum.

Our data showed the significant therapeutic effect of Nekka-Rich-containing milk replacer in calves experimentally infected with $C$. parvum. Feeding of milk replacer with Nekka-Rich reduced the severity and duration of diarrhea induced by $C$. parvum infection. In addition, isolation of $C$. parvum oocysts from feces was decreased by Nekka-Rich-containing milk replacer feeding. In fact, no oocysts were isolated $2 \mathrm{~d}$ after NekkaRich feeding begun. Furthermore, intestinal epithelial cells of calves treated with Nekka-Rich showed no adherent $C$. parvum oocysts on their surfaces, whereas epithelial cells of calves in the nontreated group showed strong C. parvum adhesion. These results indicate that C. parvum colonization in the intestines of calves infected with C. parvum oocysts can be entirely cleaned by feeding of Nekka-Rich and that calves infected with $C$. parvum can be cured by the administration of Nekka-Rich.

In this study, we showed that Nekka-Rich has a protective effect against $C$. parvum-infected calves. It has been shown that probiotics (microbes that beneficially affect the host by improving its intestinal microbial balance) such as bifidobacteria and lactic acid bacteria, are useful in treating and preventing various intestinal infections and diarrhea caused by pathogenic bacteria (Gorbach, 1990; Abe et al., 1995; Bernet-Camard et al., 1997; Bomba et al., 1999; Naidu et al., 1999; Heyman, 2000; Vold et al., 2000) and C. parvum (Alak et al., 1997, 1999). Previously, we showed that wood vinegar liquid from the bark of an evergreen oak, which is present in Nekka-Rich, has a stimulatory effect on the growth of E. faecium and Bifidobacterium thermophilum, both of which act as probiotics (Watarai and Tana, 2005). Thus, the growth-stimulating effect of wood vinegar liquid from the bark of an evergreen oak against probiotics would also be related to the removal effect of NekkaRich against $C$. parvum-infected calves. 
A new method that inhibits the infection of $C$. parvum oocysts in calves would clearly be valuable. We have found that $C$. parvum colonization of mucosal surfaces and excretion of the oocysts in feces are effectively inhibited by administration of Nekka-Rich. This can be explained by the fact that activated charcoal of NekkaRich showed effectiveness for removal of C. parvum oocysts, and also that wood vinegar liquid from the bark of an evergreen oak has a disinfectant effect against $C$. parvum oocysts. The use of Nekka-Rich as an additive would be effective in preventing $C$. parvum infection in calves, thereby facilitating eradication of the disease. Additionally, inhibition of oocyst shedding by feeding of Nekka-Rich would have a significant impact on controlling contamination of the environment and potential waterborne spread of disease.

\section{CONCLUSIONS}

Cryptosporidium parvum oocysts were adsorbed, in a dose-dependent manner, by activated charcoal of NekkaRich. In addition, wood vinegar liquid included in NekkaRich showed activity against $C$. parvum oocysts. It was shown that Nekka-Rich has an anticryptosporidial effect in calves. Although nontreated control calves showed severe diarrhea and fecal excretion of oocysts after infection with C. parvum oocysts, those fed milk replacer with Nekka-Rich showed recovery from diarrhea $1 \mathrm{~d}$ after the start of treatment, and no shedding of oocysts occurred on $2 \mathrm{~d}$ from the beginning of Nekka-Rich treatment. Thus, we have very promising preliminary data supporting the anticryptosporidial efficacy of NekkaRich in reducing or eliminating C. parvum infection in calves.

\section{REFERENCES}

Abe, F., N. Ishibashi, and S. Shimamura. 1995. Effect of administration of bifidobacteria and lactic acid bacteria to newborn calves and piglets. J. Dairy Sci. 78:2838-2846.

Alak, J. I., B. W. Wolf, E. G. Mdurvwa, G. E. Pimentel-Smith, and O. Adeyemo. 1997. Effect of Lactobacillus reuteri on intestinal resistance to Cryptosporidium parvum infection in a murine model of acquired immunodeficiency syndrome. J. Infect. Dis. 175:218221.

Alak, J. I., B. W. Wolf, E. G. Mdurvwa, G. E. Pimentel-Smith, S. Kolavala, H. Abdelrahman, and V. Suppiramaniam. 1999. Supplementation with Lactobacillus reuteri or L. acidophilus reduced intestinal shedding of cryptosporidium parvum oocysts in immunodeficient C57BL/6 mice. Cell. Mol. Biol. 45:855-863.

Anderson, B. C. 1998. Cryptosporidiosis in bovine and human health. J. Dairy Sci. 81:3036-3041.

Anderson, M. E. 1992. Efficacies of acetic, lactic and two mixed acids in reducing numbers of bacteria on surfaces of lean meat. J. Food Saf. 12:139-147.
Bernet-Camard, M. F., V. Lievin, D. Brassart, J. R. Neeser, A. L. Servin, and S. Hudault. 1997. The human Lactobacillus acidophilus strain LA1 secretes a nonbacteriocin antibacterial substance(s) active in vitro and in vivo. Appl. Environ. Microbiol. 63:2747-2753.

Bomba, A., R. Nemcova, S. Gancarcikova, R. Herich, and R. Kastel. 1999. Potentiation of the effectiveness of Lactobacillus casei in the prevention of $E$. coli induced diarrhea in conventional and gnotobiotic pigs. Adv. Exp. Med. Biol. 473:185-190.

Casemore, D. P., S. E. Wright, and R. L. Coop. 1997. Cryptosporidiosis: Human and animal epidemiology. Pages 65-92 in Cryptosporidium and cryptosporidiosis. R. Fayer, ed. CRC Press, New York, NY.

Chandy, T., and C. P. Sharma. 1998. Activated charcoal microcapsules and their applications. J. Biomater. Appl. 13:128-157.

Drucker, M. M., J. Goldhar, P. L. Ogra, and E. Neter. 1977. The effect of attapulgite and charcoal on enterotoxicity of Vibrio cholerae and Escherichia coli enterotoxins in rabbits. Infection 5:211-213.

Du, X.-N., Z. Niu, G.-Z. Zhou, and Z.-M. Li. 1987. Effect of activated charcoal on endotoxin adsorption. Part I. An in vitro study. Biomater. Artif. Cells Artif. Organs 15:229-235.

Fayer, R., U. Morgan, and S. J. Upton. 2000. Epidemiology of Cryptosporidium: Transmission, detection, identification. Int. J. Parasitol. 30:1305-1322.

Gardiner, K. R., N. H. Anderson, M. D. McCaique, P. J. Erwin, M. I. Halliday, and B. J. Rowlands. 1993. Adsorbents as antiendotoxin agents in experimental colitis. Gut 34:51-55.

Gorbach, S. L. 1990. Lactic acid bacteria and human health. Ann. Med. 22:37-41.

Heyman, M. 2000. Effect of lactic acid bacteria on diarrheal diseases. J. Am. Coll. Nutr. 19:137s-146s.

Hsiao, C. P., and K. J. Siebert. 1999. Modeling the inhibitory effects of organic acids on bacteria. Int. J. Food Microbiol. 47:189-201.

Kniel, K. E., S. S. Sumner, D. S. Lindsay, C. R. Hackney, M. D. Pierson, A. M. Zajac, D. A. Golden, and R. Fayer. 2003. Effect of organic acids and hydrogen peroxide on Cryptosporidium parvum viability in fruit juices. J. Food Prot. 66:1650-1657.

Naidu, A. S., W. R. Bidlack, and R. A. Clemens. 1999. Probiotic spectra of lactic acid bacteria (LAB). Crit. Rev. Food Sci. Nutr. 39:13-126.

Naka, K., S. Watarai, Tana, K. Inoue, Y. Kodama, K. Oguma, T. Yasuda, and H. Kodama. 2001. Adsorption effect of activated charcoal on enterohemorrhagic Escherichia coli. J. Vet. Med. Sci. 63:281-285

Nakai, S. A., and K. J. Siebert. 2003. Validation of bacterial growth inhibition models based on molecular properties of organic acids. Int. J. Food Microbiol. 86:248-255.

Pegues, A. S., S. S. Sofer, R. E. McCallum, and L. B. Hinshaw. 1979 The removal of $14 \mathrm{C}$ labeled endotoxin by activated charcoal. Int. J. Artif. Organs 2:153-158.

Smith, H. V. 1998. Detection of parasites in the environment. Parasitology 117:S113-S141.

Uga, S., J. Matsuo, E. Kono, K. Kimura, M. Inoue, S. K. Rai, and K. Ono. 2000. Prevalence of Cryptosporidium parvum infection and pattern of oocyst shedding in calves in Japan. Vet. Parasitol. 94:27-32.

Vold, L., A. Holck, Y. Wasteson, and H. Nissen. 2000. High levels of background flora inhibits growth of Escherichia coli O157:H7 in ground beef. Int. J. Food Microbiol. 56:219-225.

Watarai, S., and Tana. 2005. Eliminating the carriage of Salmonella enterica serovar Enteritidis in domestic fowls by feeding activated charcoal from the bark containing wood vinegar liquid (NekkaRich). Poult. Sci. 84:515-521.

Wicks, S. R., N. E. Richardson, and B. J. Meakin. 1980. The use of polyamide coatings for selective adsorption control on activated charcoal. J. Biomed. Mater. Res. 14:743-751.

Xiao, L., R. Fayer, U. Ryan, and S. J. Upton. 2004. Cryptosporidium taxonomy: Recent advances and implications for public health. Clin. Microbiol. Rev. 17:72-97. 